\title{
Anatomy of digestive and respiratory system of Indian grey mongoose (Herpestes edwardsii)
}

\author{
S K Shil ${ }^{1}$, B C Das ${ }^{2}$, M Uddin ${ }^{1}$, M L Rahman ${ }^{1}$, M A Quasem ${ }^{1}$. \\ ${ }^{1}$ Department of Anatomy and Histology, ${ }^{2}$ Department of Medicine and Surgery, Chittagong Veterinary and Animal \\ and Sciences University, Khulshi, Chittagong-4202
}

Mongooses (Herpestidae) are small, widespread carnivores occupying various habitats from Africa to Southeast Asia (Thulin et al., 2006). The genus Herpestes contains 10 species (Nowak, 1999) and is considered the oldest genus within the order Carnivora, dating back approximately 30 million years (Hinton and Dunn, 1967). The Indian gray mongoose or common grey mongoose (Herpestes edwardsii) is a species of mongoose mainly found in southern Asia mainly India, Pakistan, Nepal, Sri Lanka and some other parts of Asia (Choudhury et al., 2011). According to IUCN Red list 2012 (IUCN, 2012) status they are listed as Least Concern. They are terrestrial, diurnal and commonly found in open forests, scrublands and cultivated fields, often close to human habitation (Prater, 1980). This omnivorous scavenger preys on rodents, snakes, birds' eggs and hatchlings, lizards and variety of invertebrates (Choudhury et al., 2011). The Indian gray mongoose is one of the few animals that can survive a cobra attack, which makes it one of the deadly snake's few predators. As there is limited information about the anatomy of digestive and respiratory system of mongoose, this will be a platform for better understanding of physiology, pathology of diseases and subsequent application in clinical examination and surgical intervention if needed.

An adult male Indian grey mongoose (Herpestes edwardsii) was brought in Sahedul Alam Quadery teaching veterinary hospital, Chittagong for surgical treatment but it was found clinically dead. Before post mortem examination species name, body weight, body length, tail length, external features were recorded in hospital case record sheet. Keeping in dorsal recumbancy, a longitudinal incision was made at the ventral midline from pharynx to the pelvic inlet. Thus organs were examined sequentially without any damage or distortion. After thorough post mortem examination it was subjected to anatomical measurement in Anatomy laboratory of the same University. Different views were examined to describe the organs. Length of coiled portion of the respective organ was measured by thread placing on along the long axis of the tubular organ. Circumference was taken with full of content of the organ. Diameter was measured by using slide caliper (0-150 digital caliper, Shinko Denshi Co. Ltd, Japan). Tracheal length was measured from last laryngeal cartilage to the tracheal bifurcation. Each lobe of the lung was studied well by removing the loose attachment within them. For nomenclature Nomina Anatomica Veterinaria, 1994, 4th edition was used.

\section{Digestive system}

The digestive tract of Mongoose consist of oesophagus, stomach, duodenum, Jejunum, ileum, caecum, colon and rectum. Accessory digestive organs includes teeth, tongue, salivary glands, liver, pancreas. Among them liver and pancreas were studied well. Oesophagus was straight tube with a length of $12.3 \mathrm{~cm}$ and diameter at cranial and caudal portion was $6.2 \mathrm{~mm}$ and $4.7 \mathrm{~mm}$ respectively. The stomach was the carnivore type. It was ' $\mathrm{J}$ ' shaped with a total length of $9.5 \mathrm{~cm}$ and circumference at cardiac, fundic, pyloric region was 6 $\mathrm{cm}, 6 \mathrm{~cm}$ and $4 \mathrm{~cm}$ respectively (Fig.1). Duodenum starts from the pyloric sphincter and continues about 10 $\mathrm{cm}$ with a maximum diameter of $5.0 \mathrm{~mm}$. Pancreas is located along the border of the duodenum. The jejunum was the longest portion among the three parts of the small intestine which had a total length of $92 \mathrm{~cm}$ with a maximum diameter of $4.92 \mathrm{~mm}$. Unlike other carnivore they have their centrifugal and centripetal coiled portion having length of $16 \mathrm{~cm}$ with cranial and caudal continuation of ' $U$ ' shaped loops (Fig.2).

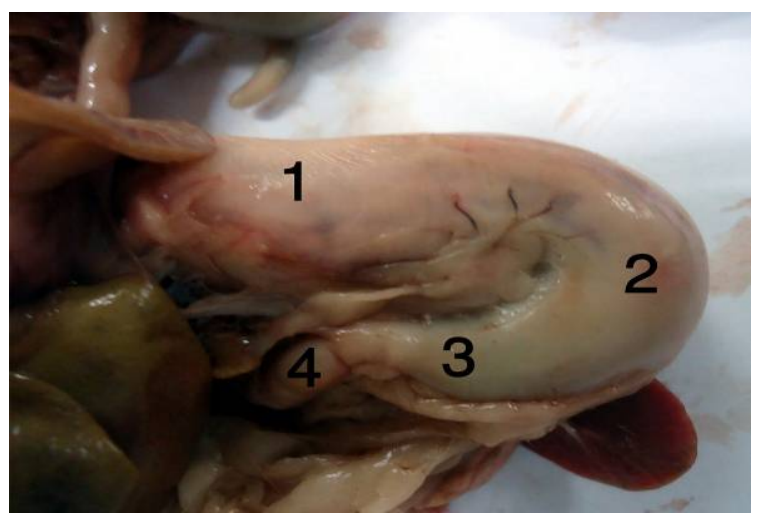

Fig. 1. Simple stomach of Indian Grey Mongoose. 1.Cardic region, 2. Fundic region, 3. Pyloric region 4. Pyloric sphincter

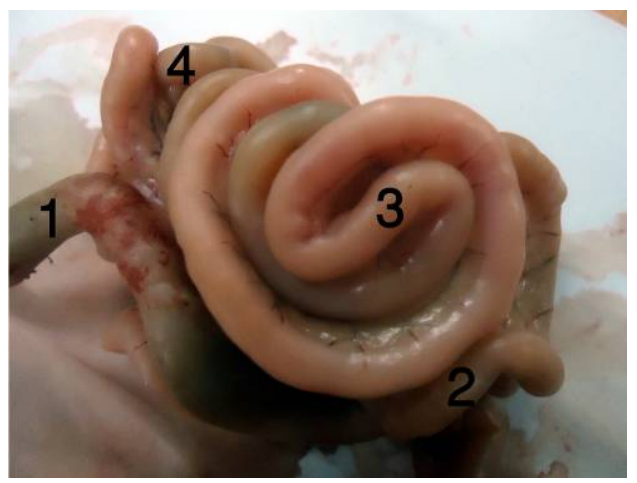

Fig. 2. Jejunum of Indian Grey Mongoose. 1.Duodenum, 2.Cranial part of Jejunum , 3.Centripetal and centrifugal coiling of Jejunum, 4.Caudal part of Jejunum. 
Coiled portion starts $14 \mathrm{~cm}$ away from the starting point of the jejunum and rest of the part holds a length of 62 $\mathrm{cm}$ with uniform diameter. In dog and cat there is no such concentric coil (Getty, 1975). Ileum, the straight portion of the small intestine has its length and diameter, $9 \mathrm{~cm}$ and $4.2 \mathrm{~mm}$ respectively. Caecum was elongated comma shaped structure situated at the junction of the ileum and colon with a length of $3.8 \mathrm{~cm}$ (Fig.3). Its diameter at base and apex was $4.0 \mathrm{~mm}$ and $3.0 \mathrm{~mm}$ respectively. Colon was straight tube with length $4.3 \mathrm{~cm}$ and diameter $5 \mathrm{~mm}$. Rectum was also the straight tube which terminates at the anus. Its maximum length was $7.1 \mathrm{~cm}$ with circumference $2.7 \mathrm{~cm}$. Liver was divided into five chief lobes and two processes by fissures which converge at the portal fissure. During examination in soft condition the lobes were spread out so as it was well visible (Fig.4).

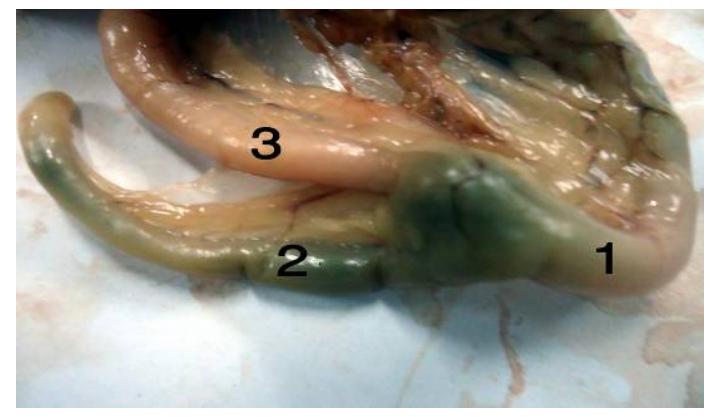

Fig. 3. Elongated comma shape caecum of Indian Grey Mongoose. 1.lleum, 2.Caecum, 3.Colon.

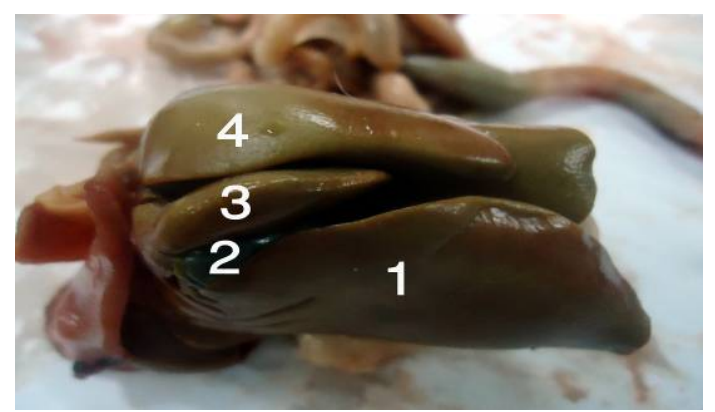

Fig. 4. Parietal surface of the liver of Indian Grey Mongoose. 1. Right medial lobe, 2.Gall Bladder, 3.Quadrate process, 4.Left medial lob.

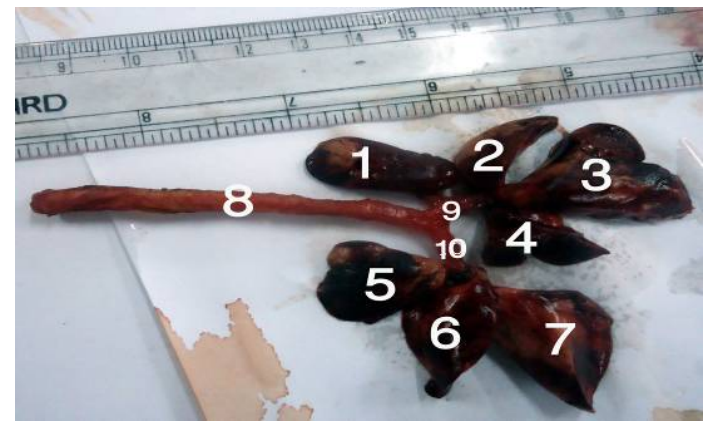

Fig. 5. Right and left lobes of lungs with trachea of mongoose.
The right medial lobe was the largest and rectangular in outline. The left lateral lobe was second in size and was somewhat tongue shaped. But the left medial lobe was third in size and was prismatic. Caudate lobe had deep impression of right kidney. In dog and cat left lateral lobe was largest and was oval in outline. The left medial lobe was smaller and prismatic. The right medial lobe was second in size and was somewhat tongue shaped (Getty, 1975). Usually it does not reach to the ventral border of the liver. It was visible well from the parietal surface of the liver (Fig.4). Pancreas was located along the border of the duodenum with a length of $15 \mathrm{~cm}$.

\section{Respiratory system}

Respiratory system of mongoose consists of nasal cavity, larynx, trachea and lungs. Trachea was $8 \mathrm{~cm}$ long tube consisted of incomplete ' $C$ ' shaped cartilaginous ring in which incomplete part meet face to face at the dorsal aspect. The cranial and caudal part was $5.16 \mathrm{~mm}$ and $4.60 \mathrm{~mm}$ diameter respectively. Trachea bifurcates into right and left principal bronchi. Diameter of right and left principal bronchi at their origin was $4.42 \mathrm{~mm}$ and $3.64 \mathrm{~mm}$ respectively. The right apical lobar bronchus originated from $2.30 \mathrm{~mm}$ away from the tracheal bifurcation whereas left apical lobar bronchus was originated $4.46 \mathrm{~mm}$ away from the bifurcation. Right lung possess four lobes namely apical (cranial), middle (cardiac), diaphragmatic (caudal) and accessory (intermediate) lobes whereas left lung possess 3 lobes namely apical (cranial), middle (cardiac) and diaphragmatic (caudal) lobe (Fig.5). Right lung was larger than the left. Right apical lobe originated more craniad than the left. All the lobes were well separated. In left lung there had no distinct cardiac notch as in dog (Miller et al., 1964). Accessory lobe was conical in shape with concave basal border.

\section{References}

Choudhury, A., Wozencraft, C., Muddapa, D., Yonzon, P., Jennings, A. \& Geraldine, V. 2011. Herpestes edwardsii. In: IUCN 2012. IUCN Red List of Threatened Species. Version 2012.1. www.iucnredlist.org.

Getty, R. 1975. Carnivore Digestive System. In: Sisson and Grossman's The Anatomy of the Domestic Animals, $5^{\text {th }}$ edn. Vol 2. W. B. Saunders Company. Philadelphia, USA. 1547-1557 .

Hinton, H.E \& Dunn, A.M.S. 1967. Mongooses: Their Natural History \& Behavior. London: Oliver \& Boyd Ltd.

IUCN, 2012. IUCN Red List of Threatened Species (ver. 2012.1). Available at: http://www.iucnredlist.org.

Miller, M.E., Christensen, G.C \& Evans, H.E. 1964. Carnivore Respiratory System. In: Anatomy of The Dog. $1^{\text {st }}$ edn. W. B. Saunders Company, Philadelphia, USA. 734-738.

Nomina Anatomica Veterinaria, 1994. $4^{\text {th }}$ edn. Copyright by the World Association of Veterinary Anatomists. Printed in the USA.

Nowak, R.M. 1999. Carnivora; Family Herpestidae. In: Walker's Mammals of the World, $6^{\text {th }}$ edn. Vol. 1. The John Hopkins University Press, Baltimore, London. 766-786.

Thulin, C.G., Simberloff, D., Barun, A., Pascal, M., \& Islam, M.A. 2006. Genetic divergence in the small Indian mongoose (Herpestes auropunctatus), a widely distributed invasive species. Molecular Ecology. 15: 3947-3956. 